\title{
Can sedation using a combination of propofol and dexmedetomidine enhance the satisfaction of the endoscopist in endoscopic submucosal dissection?
}

\section{(๑) $\odot \ominus$}

\author{
Authors \\ Takashi Nonaka1,2, Masahiko Inamori ${ }^{3}$, Tetsuya Miyashita ${ }^{4}, Y^{1}$ umi Inoh², Kenji Kanoshima², Takuma Higurashi², \\ Hidenori Ohkubo², Hiroshi lida ${ }^{3}$, Koji Fujita ${ }^{5}$, Akihiko Kusakabe ${ }^{5}$, Takahisa Gotoh ${ }^{4}$, Atsushi Nakajima'
}

Institutions

1 Department of Gastroenterology, National Hospital Organization Yokohama Medical Center, Yokohama, Japan

2 Department of Gastroenterology and Hepatology, Yokohama City University School of Medicine, Yokohama, Japan

3 Department of Medical Education, Yokohama City University School of Medicine, Yokohama, Japan

4 Department of Anesthesiology, Yokohama City University School of Medicine, Yokohama, Japan

5 Office of Postgraduate Medical Education, Yokohama City University Hospital, Yokohama, Japan

submitted 10.5.2017

accepted after revision 26.9.2017

Bibliography

DOI https://doi.org/10.1055/s-0043-122228 |

Endoscopy International Open 2018; 06: E3-E10

(c) Georg Thieme Verlag KG Stuttgart · New York

ISSN 2364-3722

Corresponding author

Masahiko Inamori, MD, PhD, Department of Medical

Education, Yokohama City University School of Medicine,

3-9 Fukuura, Kanazawa-ku, Yokohama, 236-0004, Japan

Fax: +81-45-784-3546

inamorim@yokohama-cu.ac.jp

\section{ABSTRACT}

Background and study aims The aim of this pilot randomized controlled trial was to evaluate and compare the satisfaction of the endoscopist along with the effectiveness and safety of sedation between sedation protocol using a combination of propofol (PF) and dexmedetomidine (DEX) (Combination group) and sedation protocol using PF alone (PF group) during gastric endoscopic submucosal dissection (ESD).

Patients and methods Fifty-eight patients with gastric neoplasias scheduled for gastric ESD were enrolled and randomly assigned to the two groups. The satisfaction scores of the endoscopists and the parameters for the effectiveness and safety of sedation were evaluated by comparisons between the two groups.

Results The satisfaction scores of the endoscopists, which were measured using a visual analogue scale, were significantly higher in the Combination group than in the PF group ( 88 vs. $69, P=0.003$ ). The maintenance dose of $P F$ was lower in the Combination group than in the PF group $(2 \mathrm{mg} / \mathrm{kg} / \mathrm{h}$ vs. $5 \mathrm{mg} / \mathrm{kg} / \mathrm{h}, P<0.001)$, and the number of rescue $P F$ injections was fewer in the Combination group than in the PF group ( 2 times vs. 6 times, $P<0.001$ ). The incidence of bradycardia (defined as a pulse rate $\leq 45 \mathrm{bpm}$ ) in the Combination group was higher than that in the PF group $(37.9 \%$ vs. $10.3 \%, P=0.029)$.

Conclusions This study suggests that gastroenterologistdirected sedation using a combination of PF and DEX during gastric ESD can enhance the satisfaction levels of endoscopists by providing stable sedation with an acceptable safety profile.

\section{Introduction}

Endoscopic submucosal dissection (ESD) can provide complete resection of even large lesions in an en bloc manner and allow precise assessment of the histopathological curability of resected specimens. This procedure also reduces postoperative loss of function. Therefore, ESD is currently recognized as a standard treatment for early-stage gastrointestinal carcinomas, which have an extremely low risk of lymph node metastasis [1]. However, ESD requires complicated and time-consuming manoeuvers and occasionally causes life-threatening complications $[2,3]$. Therefore, adequate sedation during the procedure is mandatory to achieve successful completion of ESD.

Recently, propofol (PF) has been increasingly used in many gastrointestinal endoscopic procedures worldwide [4]. A large number of studies have also been published supporting the 
usefulness of sedating patients using PF alone during ESD because it achieves better outcomes, including less restlessness and quicker recovery times, and has a safety profile that is similar to conventional sedation with midazolam (MDZ) [5-7]. More recently, the introduction of dexmedetomidine (DEX) has attracted a considerable amount of attention for its usefulness in sedation during ESD [8,9]. However, there are currently no clearly established guidelines regarding the most effective and safe protocols for ESD or who the sedatives should be administered by. In addition, little is known about the endoscopist's satisfaction regarding the sedation method used during ESD.

We developed a sedation protocol using a combination of PF and DEX for ESD, with the hypothesis that this approach would provide stable and safe sedation and improve the endoscopist's ease of manipulation during ESD. We previously demonstrated the efficacy and safety of sedation under the guidance of anaesthesiologists using a combination of PF and DEX during oesophgeal ESD compared with conventional sedation using MDZ [10]. However, our previous report did not demonstrate superiority, and additional effects from the addition of DEX to PF were not detected because no comparative analysis between PF alone and a combination of PF and DEX was performed; moreover, that study had the limitation of being retrospective. This pilot randomized trial was conducted to evaluate and compare the satisfaction of the endoscopist, along with the effectiveness and the safety of sedation, between sedation using a combination of PF and DEX under the guidance of gastroenterologists and sedation using PF alone during gastric ESD.

\section{Patients and methods}

\section{Patients}

Fifty-eight patients with gastric neoplasias (68 lesions) scheduled for ESD at Yokohama City University Hospital during the period from April 2015 through March 2016 were enrolled and randomly assigned to a sedation protocol using PF alone (PF group, 29 patients) or a sedation protocol using a combination of PF and DEX (Combination group, 29 patients) according to a random number list. An investigator who was not directly involved in this study preorderd a random number list using Excel 2013 (Microsoft Co., Redmond, WA., USA) and was responsible for the allocation and concealment.

Patients classified with an American Society of Anesthesiologist (ASA) physical status higher than II, patients with a tracheotomy orifice or a cardiac pacemaker, patients with a history of allergy to the study drugs, and patients without written informed consent were excluded.

\section{Medications and sedation protocol}

All patients received supplemental oxygen at a flow rate of $2 \mathrm{~L} /$ min via nasal cannula. Local pharyngeal anaesthesia was performed using an $8 \%$ topical lidocaine spray prior to the intravenous administration of the sedatives in all cases. ESD procedures were performed in the endoscopy laboratory by four skilled endoscopists accredited by the Japan Gastroenterological Endoscopy Society. Endoscopists with a minimum experience of performing more than 100 cases of gastric ESD participated
- Table 1 Modified Observer's Assessment of Alertness/Sedation Scale (MOSS/A).

\begin{tabular}{|l|l|}
\hline Score & Responsiveness \\
\hline 6 & Agitated \\
\hline 5 & Responds readily to name spoken in normal tone \\
\hline 4 & Lethargic response to name spoken in normal tone \\
\hline 3 & Responds only after name is called loudly and/or repeatedly \\
\hline 2 & Responds only after mild prodding or shaking \\
\hline 1 & Does not respond to mild prodding or shaking \\
\hline 0 & Does not respond to deep stimulus \\
\hline
\end{tabular}

in the present study. All sedation protocols were performed by two gastroenterologists who were not directly involved in the endoscopic procedure. The gastroenterologists in charge of sedation were well trained in basic and advanced cardiac life support before participating in this study and administered the study drugs under the supervision of anaesthesiologists. Anaesthesiologists were also on standby in case of an emergency. Resuscitation equipment was available in the endoscopy laboratory at all times. The target level of sedation during ESD was deep sedation, namely, a score of 1 or 2 on the Modified Observer's Assessment of Alertness/Sedation Scale (MOAA/S) [11] ( Table 1).

In the PF group, an initial bolus of $20 \mathrm{mg}$ of PF (Diprivan; AstraZeneca K.K., Osaka, Japan) was administered intravenously for induction, and the bolus administration of $20 \mathrm{mg}$ doses of PF was repeated until the patients reached the proper sedation level, which was reflected by the loss of the eyelash reflex. After induction, the continuous infusion of PF at $2 \mathrm{mg} / \mathrm{kg} / \mathrm{h}$ was used for maintenance ( $>$ Fig. 1 ).

In the Combination group, given simultaneously with the same protocol for PF administration as the PF group, a continuous-rate infusion of DEX (Precedex; Hospira Japan Co., Ltd., Osaka, Japan) was performed using a rate of $6 \mu \mathrm{g} / \mathrm{kg} / \mathrm{h}$ for $10 \mathrm{~min}$ to induce sedation. This was followed by the continuous administration of DEX at fixed rate of $0.5 \mu \mathrm{g} / \mathrm{kg} / \mathrm{h}$ as the maintenance infusion rate ( $>$ Fig. 2 ).

For both groups, a rescue bolus injection of $20 \mathrm{mg}$ of PF was administered if the patient began to move or show signs of discomfort during ESD. If the MOAA/S scale showed a score of three or more, the $\mathrm{PF}$ infusion rate for maintenance, in not only the PF group but also the Combination group, was increased by $1 \mathrm{mg} / \mathrm{kg} / \mathrm{h}$ in a stepwise manner. In contrast, the maintenance infusion rate of DEX in the Combination group remained fixed until the removal of the lesion.

\section{Monitoring}

Electrocardiography, an automated blood pressure metre, pulse oximetry, and capnography were used for intraoperative monitoring in all cases. In addition, consciousness levels were assessed initially after the induction of sedation and then at 15-min intervals thereafter during ESD using the MOAA/S scale. All monitoring information andadministered medications were 
PF $20 \mathrm{mg}$

$\mathrm{PF} 2 \mathrm{mg} / \mathrm{kg} / \mathrm{h}$

(dose escalation based on sedation level)

$\nabla \nabla \nabla \bullet$

$\underbrace{}_{\text {Induction phase }}$

Maintenance phase

- Fig. 1 Sedation protocol in the PF group.

PF $20 \mathrm{mg}$

$\mathrm{PF} 2 \mathrm{mg} / \mathrm{kg} / \mathrm{h}$

(dose escalation based on sedation level)

$\nabla \nabla \nabla \vee \bullet$

Induction phase

Maintenance phase

DEX $6 \mu \mathrm{g} / \mathrm{kg} / \mathrm{h}$ for $10 \mathrm{~min}$. DEX $0.5 \mu \mathrm{g} / \mathrm{kg} / \mathrm{h}$ (fixed infusion rate)

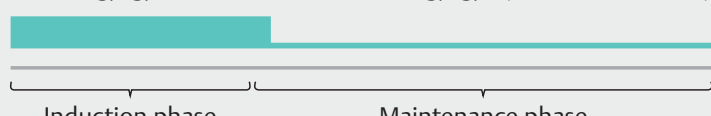

Induction phase

Maintenance phase

Fig. 2 Sedation protocol in the Combination group.

recorded in the anaesthesia chart by a gastroenterologist in charge of sedation.

\section{Outcome measurement and definitions}

The clinical characteristics of the patients (including age, gender, body mass index, Brinkman index, alcohol consumption, underlying diseases, and ASA physical status), baseline vital signs (including percutaneous oxygen saturation, systolic blood pressure and pulse rate), and endoscopic findings for gastric neoplasms (including the lesion localized site and macroscopic type) were obtained before ESD. In addition, histopathological findings after ESD (including invasion depth of the lesion, the coexistence of an ulcer scar and the resected specimen size) and ESD outcomes (including en bloc resection rate, complete resection rate, total sedation times, procedural times for resection and procedure-related complications) were collected. Administered medications, patient monitoring information during ESD and recovery times were assessed from the anaesthesia chart. At the end of ESD, endoscopists and sedation providers scored the degree of their satisfaction based on the performance of the procedure and the stability of sedating the patients using a visual analogue scale (VAS) ranging from 0 (most unsatisfactory) to 100 (most satisfactory).

We defined presence of hypertension, diabetes mellitus, dyslipidemia, cerebral infarction, ischemic heart disease, pulmonary disease, liver cirrhosis and renal dysfunction as underlying diseases. Pulmonary disease was defined as asthma, chronic obstructive pulmonary disease, bronchiectasis, sequelae of pulmonary tuberculosis or tuberculous pleurisy and interstitial lung disease. Renal dysfunction was defined as an estimated glomerular filtration rate (eGFR) of less than $60 \mathrm{ml} / \mathrm{min} /$
$1.73 \mathrm{~m}^{2}$ [12]. Localized site and invasion depth of the lesion were classified according to the Japanese classification of gastric cancer by the Japanese Gastric Cancer Association [13]. The macroscopic type was classified as either elevation or depression. A flat lesion was included in depression. En bloc resection was defined as resection in a single piece. Complete resection was defined as tumour-free lateral and vertical margins on histopathology in en bloc resected specimens. Procedural times for resection, which were defined as the duration from the starting incision of the mucosa to complete removal, were recorded with a digital video recorder. Procedure-related complications were evaluated as the occurrence of bleeding and perforation. Bleeding was defined as intraoperative bleeding that required a blood transfusion, bleeding symptoms such as haematemesis or melena after returning to the ward, or a decrease of greater than $2 \mathrm{~g} / \mathrm{dL}$ in haemoglobin levels according to a haematological test performed on the day after ESD. A diagnosis of perforation required direct endoscopic visualization of mesenteric fat or radiographic evidence of free air on a computed tomography scan. Recovery times was defined as the duration from the cessation of all sedatives administration to the conscious state in which a patient can follow the physician's instructions.

\section{Study endpoints}

The primary endpoint of this study was to compare the satisfaction score of the endoscopists between the PF group and the Combination group. In addition, the effectiveness and the safety of sedation were assessed as secondary endpoints. The parameters for the effectiveness of sedation were defined as the following: "the degree of satisfaction of the sedation providers" performing the management of sedatives and the sedation level, "the maintenance dose of PF" required to maintain proper sedation level, "the number of rescue PF injections" required to prevent patient body movements and discomfort, and the presence or absence of "restlessness" defined as severe patient body movements requiring physical restraint. The parameters for the safety of sedation adopted the presence or absence of "hypoxemia", which was defined as a percutaneous oxygen saturation level $\leq 94 \%$; "hypotension", which was defined as a systolic blood pressure $\leq 80 \mathrm{mmHg}$; "bradycardia”, which was defined as a pulse rate $\leq 45$ beats per minute (bpm); and "serious adverse events", which were defined as those resulting in cardiorespiratory instability that cannot be immediately resolved by sedation providers alone and forced the discontinuation of ESD. These parameters were evaluated by comparisons between the two groups.

\section{Statistical analysis}

We used Fisher's exact test to perform a categorical comparison of the data. Differences between continuous data were compared using the Mann-Whitney $U$ test. The level of significance was set at a $P$-value $<0.05$. All statistical analyses were performed using IBM SPSS Statistics 22 (IBM Co., Chicago, IL, USA). 


\section{Ethics}

The current study was conducted in accordance with the Declaration of Helsinki. This study protocol was approved by the institutional review board of Yokohama City University Hospital and registered at http://www.umin.ac.jp/ctr/index/htm (registration number UMIN000013739 under UMIN Clinical Trials Registry). Written informed consent was obtained from all participants, not only for the endoscopic treatment under each sedation protocol but also for the use of the patients' clinical data for research purposes.

\section{Results}

\section{Clinical details of the patients, lesions and procedures}

Clinical details of the patients, lesions and procedures for both groups are shown in $\mathbf{T}$ Table 2 . There were no significant differences between the two groups in clinical patient characteristics, endoscopic findings for gastric neoplasias, ESD outcomes and recovery times. However, histopathological findings after ESD showed that the number of gastric neoplasias invading the submucosa was larger in the Combination group than in the PF group (5 vs. $0, P=0.019$ ).

\section{The satisfaction of the endoscopists and the parameters for the effectiveness of sedation}

- Table 3 shows the satisfaction scores of the endoscopists and the parameters for the effectiveness of sedation in both groups. The satisfaction scores of both the endoscopists and the sedation providers were significantly higher in the Combination group than in the PF group (88 [6-100] mm vs. 69 [31-96] mm, $P=0.003$, and 95 [24-100] mm vs. 67 [15-93] $\mathrm{mm}, P<0.001$, respectively, median [range]). The maintenance dose of $\mathrm{PF}$ was less in the Combination group than in the PF group $(2[2-4] \mathrm{mg} / \mathrm{kg} / \mathrm{h}$ vs. 5 [3-7] mg/kg/h, $P<0.001$, median [range]), and the number of times a rescue PF injection was needed was also fewer in the Combination group than in the PF group (2 [0-16] times vs. 6 [3-13] times, $P<0.001$, median [range]). The percentage of patients who showed restlessness was lower in the Combination group than in the PF group; however, there was no statistically significant difference between the two groups ( $6.9 \%$ vs. $24.1 \%, P=0.144)$.

\section{Parameters for safety of sedation}

- Table 4 shows the parameters for the safety of sedation that were monitored during sedation in both groups. Although the minimum percutaneous oxygen saturation level was statistically higher in the Combination group than in the PF group (96 [79-99]\% vs. 95 [68-98]\%, $P=0.029$, median [range]), there were no significant differences in the incidence of hypoxemia (defined as percutaneous oxygen saturation level $\leq 94 \%$ ) between the two groups $(24.1 \%$ vs. $34.5 \%, P=0.565)$. On the other hand, the minimum pulse rate was significantly lower in the Combination group than in the PF group (47 [34-61] bpm vs. 59 [36-72] bpm, $P<0.001$, median [range]), and the incidence of bradycardia (defined as pulse rate $\leq 45 \mathrm{bpm}$ ) was sig- nificantly higher in the Combination group than in the PF group (37.9\% vs. $10.3 \%, P=0.029$ ). Although some patients experienced sedation-related adverse events as mentioned above, hypoxemia was successfully treated by securing the airway using the jaw-thrust manoeuver or increasing supplemental oxygen; cardiovascular depression was also immediately reversed by administering medicine such as ephedrine or atropine. In the present study, no serious adverse events necessitated intubation or ventilation, and discontinuation of the procedure was not required in any cases.

\section{Discussion}

Endoscopists need to pay close attention to the occurrence of procedure-related complications resulting from patients' body movements during ESD and are therefore subjected to psychological stress and strain over a long time period. A stable sedation with few patient body movements is mandatory to enhance the swiftness and precision of the procedure and reduce the mental burden on endoscopists, leading to enhancement of the satisfaction levels of endoscopists. Many studies have been published on the usefulness of sedation for ESD using new sedatives, such as PF and DEX [5-8]. However, the evidence on sedation is limited regarding the satisfaction of endoscopists. This trial showed that sedation using PF combined with DEX enhanced the satisfaction levels of the endoscopists in comparison with sedation using PF alone.

What additional factors are important for sedation in ESD? In our opinion, the ability of the sedatives to maintain a proper sedation level that minimizes patient body movements is one of the most important features of the sedation protocol for ESD. Therefore, we evaluated that the maintenance dose of PF, the number of rescue PF injections, and the presence of restlessness as parameters of sedation effectiveness in the present study. Both the maintenance dose of PF and the number of rescue PF injections were lower in the Combination group than in the PF group. The reduction of such parameters represents sedation of high effectiveness while maintaining proper a sedation level. However, there were no statistically significant differences between the two groups in the incidence of restlessness, which was defined as "severe" body movements of the patient requiring physical restraint.

The other important factor required in sedation for ESD is safety, as the procedure should have a minimal influence on the patient's cardiorespiratory status. The definition of criteria for cardiorespiratory adverse events was determined by consensus among anaesthesiologists, endoscopists and sedation providers, which was designed for the security of patients under sedation. With respect to desaturation, setting the cut-off value of desaturation at a percutaneous oxygen saturation level of $\leq 94 \%$ was considered appropriate for the patients receiving supplemental oxygen to readily detect hypoventilation, because the measurement of oxygen saturation had been reported to be insensitive to the detection of hypoventilation [14]. A systolic blood pressure $\leq 80 \mathrm{mmHg}$ used as the cut-off value of hypotension was little debatable from the perspective of cardiovascular function. Lastly, previous literatures had reported the 
- Table 2 Clinical details of the patients, lesions and procedures.

\begin{tabular}{|c|c|c|c|}
\hline & $\begin{array}{l}\text { Combination group } \\
n=29\end{array}$ & $\begin{array}{l}\text { PF group } \\
n=29\end{array}$ & $P$-value \\
\hline Number of patients, $\mathrm{n}$ & 29 & 29 & \\
\hline Age, years & $71(52-86)$ & $74(60-86)$ & 0.196 \\
\hline Gender, n & & & 1 \\
\hline Male & 24 & 23 & \\
\hline Female & 5 & 6 & \\
\hline $\mathrm{BMI}, \mathrm{kg} / \mathrm{m}^{2}$ & $22.4(15.6-27.6)$ & $23.1(18.2-27.8)$ & 0.294 \\
\hline Brinkman index & $640(0-1800)$ & $510(0-2100)$ & 0.356 \\
\hline Alcohol consumption, g/day & $15(0-120)$ & $15(0-80)$ & 0.945 \\
\hline Underlying diseases, n, [\%] & $21[72.4]$ & $21[72.4]$ & 1 \\
\hline Hypertension & 14 & 9 & \\
\hline Diabetes mellitus & 3 & 4 & \\
\hline Dyslipidemia & 11 & 9 & \\
\hline Cerebral infarction & 2 & 2 & \\
\hline Ischaemic heart disease & 1 & 2 & \\
\hline Pulmonary disease & 2 & 3 & \\
\hline Liver cirrhosis & 0 & 0 & \\
\hline Renal dysfunction & 10 & 12 & \\
\hline ASA physical status, $\mathrm{n}$ & & & 1 \\
\hline I & 5 & 5 & \\
\hline II & 24 & 24 & \\
\hline Baseline $\mathrm{SpO}_{2}, \%$ & $97(95-100)$ & $98(94-100)$ & 0.685 \\
\hline Baseline SBP, mmHg & $145(110-199)$ & $141(100-197)$ & 0.451 \\
\hline Baseline HR, bpm & $64(51-89)$ & $71(48-95)$ & 0.082 \\
\hline Number of lesions, $\mathrm{n}$ & 32 & 36 & \\
\hline Localized site, $n$ & & & 0.479 \\
\hline Upper third & 4 & 6 & \\
\hline Middle third & 9 & 14 & \\
\hline Lower third & 19 & 16 & \\
\hline Macroscopic type, $n$ & & & 0.595 \\
\hline Elevation & 8 & 12 & \\
\hline Depression & 24 & 24 & \\
\hline Invasion depth, n & & & 0.019 \\
\hline Mucosa & 27 & 36 & \\
\hline Submucosa & 5 & 0 & \\
\hline The coexistence of ulcer scar, n, [\%] & $5[15.6]$ & $4[11.1]$ & 0.725 \\
\hline Specimen size, mm & $37.5(17-61)$ & $38.5(18-87)$ & 0.762 \\
\hline En bloc resection, n, [\%] & $32[100]$ & 35 [97.2] & 1 \\
\hline Complete resection, n, [\%] & $29[90.6]$ & 35 [97.2] & 0.336 \\
\hline
\end{tabular}


- Table 2 (Continuation)

\begin{tabular}{|c|c|c|c|}
\hline & $\begin{array}{l}\text { Combination group } \\
n=29\end{array}$ & $\begin{array}{l}\text { PF group } \\
n=29\end{array}$ & $P$-value \\
\hline Total sedation times, min & $91(32-352)$ & $118(51-455)$ & 0.086 \\
\hline Procedural times for resection, min & $39(5-304)$ & $41(11-405)$ & 0.589 \\
\hline Procedural-related complications, n, [\%] & $1[3.4]$ & $3[10.3]$ & 0.611 \\
\hline Bleeding & $0[0]$ & $2[6.9]$ & \\
\hline Perforation & $1[3.4]$ & $1[3.4]$ & \\
\hline Recovery times, min & $7(3-23)$ & $5(3-20)$ & 0.097 \\
\hline \multicolumn{4}{|c|}{$\begin{array}{l}\text { All values represent median values (range). } \\
\text { ASA, American Society of Anaesthesiologists; BMI, Body mass index; BI, Brinkman Index; PF, propofol. }\end{array}$} \\
\hline & $\begin{array}{l}\text { Combination group } \\
n=29\end{array}$ & $\begin{array}{l}\text { PF group } \\
n=29\end{array}$ & $P$-value \\
\hline \multicolumn{4}{|l|}{ Satisfaction scores, mm } \\
\hline Endoscopists & $88(6-100)$ & $69(31-96)$ & 0.003 \\
\hline Sedation providers & $95(24-100)$ & $67(15-93)$ & $<0.001$ \\
\hline \multicolumn{4}{|l|}{ Total infusion dose } \\
\hline $\mathrm{PF}, \mathrm{mg}$ & $277(146-988)$ & $584(257-2346)$ & $<0.001$ \\
\hline DEX, $\mu \mathrm{g}$ & $79(59.5-188)$ & - & - \\
\hline Maintenance dose of PF, mg/kg/h & $2(2-4)$ & $5(3-7)$ & $<0.001$ \\
\hline The number ofrescue PF injections, $n$ & $2(0-16)$ & $6(3-13)$ & $<0.001$ \\
\hline Restlessness, n, [\%] & $2[6.9]$ & $7[24.1]$ & 0.144 \\
\hline
\end{tabular}

- Table 4 Parameters for sedaton safety.

\begin{tabular}{|c|c|c|c|}
\hline & $\begin{array}{l}\text { Combination group } \\
n=29\end{array}$ & $\begin{array}{l}\text { PF group } \\
n=29\end{array}$ & $P$-value \\
\hline Minimum $\mathrm{SpO}_{2}, \%$ & $96(79-99)$ & $95(68-98)$ & 0.029 \\
\hline Hypoxemia $\left(\mathrm{SpO}_{2} \leq 94 \%\right), \mathrm{n},[\%]$ & $7[24.1]$ & $10[34.5]$ & 0.565 \\
\hline Minimum SBP, mmHg & $88(69-146)$ & $86(55-112)$ & 0.384 \\
\hline Hypotension (SBP $\leq 80 \mathrm{mmHg}$ ), n, [\%] & $12[41.4]$ & $11[37.9]$ & 1 \\
\hline Minimum PR, bpm & $47(34-61)$ & $59(36-72)$ & $<0.001$ \\
\hline Bradycardia (PR $\leq 45$ bpm), n, [\%] & 11 [37.9] & $3[10.3]$ & 0.029 \\
\hline Serious adverse events, $n,[\%]$ & $0[0]$ & $0[0]$ & - \\
\hline
\end{tabular}


criteria for bradycardia with the definition varying from a pulse rate of $<50 \mathrm{bpm}$ to $<40 \mathrm{bpm}$. Additionally, the modified Vasovagal Syncope International Study states that one standard for bradycardia requiring medical intervention is a pulse rate of $<40$ bpm [15]. Based on these, we defined the cut-off value of bradycardia as a pulse rate $\leq 45 \mathrm{bpm}$ to detect and treat immediately.

The current study demonstrated that the incidence of bradycardia in patients under sedation using a combination of PF and DEX was higher than that in patients under sedation using PF alone. DEX possesses the potential problem of cardiovascular suppression by suppressing the release of norepinephrine from sympathetic nerve endings and weakening the sympathetic nerve activity [16-18]. However, a previous meta-analysis of randomized controlled trials comparing DEX with MDZ for gastrointestinal endoscopy showed that there were no significant differences in the incidence of bradycardia between sedation using DEX and using MDZ [19]. Compared to the effects of using DEX alone, the synergistic effects of PF and DEX may have affected the incidence of bradycardia at the present study. In addition, previous research has demonstrated that cardiovascular suppression is frequently observed when using a loading infusion of DEX [20]. Our protocol for DEX administration may have also influenced the incidence of bradycardia in the present study.

The current study showed that minimum values of vital signs during sedation were very low in both groups. It is unkown whether these are much lower compared to the results in the past literatures because few previous researches showed the lowest values of vital signs during sedation. One of the reasons for the low degree of our result values might be the target level of our sedation protocol, namely, deep sedation. When adverse events occurred, well trained sedation providers promptly initiated airway management for respiratory depression or pharmacologic intervention for cardiovascular depression. Consequently, all cardiorespiratory events were immediately reversed, and therefore the minimum values of vital signs were transient. Regardless of the degree of the minimum values of vital signs, the persistent cardiorespiratory instability, which cannot be immediately resolved after performing the abovementioned procedures and requires the interruption of ESD to provide intensive care, is "true" serious adverse events. We defined such situations as serious adverse events and observed no case in which ESD had to be interrupted as a result of cardiorespiratory depression in the present study. The high satisfaction scores of the sedation providers in the Combination group may represent the safety of our protocol. However, the number of facilities that are capable of assigning a dedicated sedation provider is not so much. Therefore, the results of the present study should be carefully interpreted.

With respect to a sedation protocol using PF alone, Kiriyama $S$, et al. previously demonstrated that the starting level of continuous infusion rate of $\mathrm{PF}$ at $3 \mathrm{mg} / \mathrm{kg} / \mathrm{h}$ for maintenance was appropriate dose in the efficacy and safety of sedation during gastric ESD [5]. The maintenance infusion rate of PF in our protocol was lower than that in their report. In addition, an analgesic agent such as pentazocine or pethidine hydrochloride was not used in conjunction with sedation. Unlike PF, DEX possesses an analgesic property in addition to its anxiolytic and hypnotic activities. Although the usefulness of concurrently used analgesics have not yet been sufficiently investigated for deep sedation [21], analgesics are generally used with PF during ESD in clinical practice. These differences compared with previously reported studies may have influenced the results of the current study.

Furthermore, this trial with gastroenterologist-directed sedation included the only patients classified with an ASA physical status I or II based on the American Society for Gastrointestinal Endoscopy (ASGE) Task Force recommendation that anaesthesiologists should be considered when providing sedation for ASA physical status III, IV, and V patients during endoscopy [14]. However, ESD and sedation are sometimes applied in high risk patients with severe comorbidity, and these patients might have more severe sedation-related adverse events under sedation using a combination of PF and DEX. The exclusion of patients classified with an ASA physical status higher than II may have influenced the results of the present study, which might limits the generalizability of our protocol.

In addition to the above, there are several limitations to this study. First, the results are from a single-centre trial. Therefore, generalization of the results is difficult, and an additional multicentre trial is necessary. Second, our study sample size was not transcendentally determined due to a lack of a priori knowledge. The limited statistical power might have impacted the exclusion of severe adverse events or clarification of the effectiveness and safety of sedation using a combination of PF and DEX. Based on this pilot study, further study with adequate statistical power must be conducted. Third, when selecting a sedation protocol, medical cost is an important factor. According to the standard price of medicine prescribed under the Japanese Health Insurance System in 2017, the cost of PF is JPY 1920 (USD 16.82) for $500 \mathrm{mg} / \mathrm{vial}$ and that of DEX is JPY 5122 (USD 44.87 ) for $200 \mu \mathrm{g} /$ vial. The cost-effectiveness of our sedation protocol remains in doubt. Finally, we could not apply a double-blind design because only the patients were blinded to the group allocation, while the medical staff could not be blinded. Sedation providers definitely need to know what sedatives are, taking the security of patients into consideration. However, endoscopists ought to have been blinded to the sedation methods somehow or other. Consequently, we could not completely eliminate the bias of personal preference when they graded the degree of their satisfaction.

\section{Conclusion}

Despite its limitations, this pilot randomized controlled trial suggests that sedation using a combination of PF and DEX administered by gastroenterologists during gastric ESD can enhance the satisfaction levels of endoscopists by providing stable sedation with an acceptable safety profile when combined with careful monitoring to detect bradycardia. We therefore expect that sedation using a combination of PF and DEX will be more commonly used during gastric ESD and will be further validated for its appropriateness and effectiveness. 


\section{Acknowledgements}

The authors acknowledge the help of medical stuff at Yokohama City University Hospital Endoscopy Center.

\section{Competing interets}

None

\section{References}

[1] Japanese Gastric Cancer Association. Japanese gastric cancer treatment guidelines 2010 (ver. 3). Gastric Cancer 2011; 14: 113-123

[2] Yoo JH, Shin SJ, Lee KM et al. Risk factors for perforations associated with endoscopic submucosal dissection in gastric lesions: emphasis on perforation type. Surg Endosc 2012; 26: 2456 - 2464

[3] Toyokawa T, Inaba T, Omote $S$ et al. Risk factors for perforation and delayed bleeding associated with endoscopic submucosal dissection for early gastric neoplasms:analysis of 1123 lesions. J Gastroenterol Hepatol 2012; 27: 907-912

[4] Qadeer MA, Vargo J], Khandwala F et al. Propofol versus traditional sedative agents for gastrointestinal endoscopy: A meta-analysis. Clin Gastroenterol Hepatol 2005; 3: 1049-1056

[5] Kiriyama S, Gotoda T, Sano H et al. Safe and effective sedation in endoscopic submucosal dissection for early gastric cancer: a randomized comparison between propofol continuous infusion and intermittent midazolam injection. J Gastroenterol 2010; 45: 831-837

[6] Sasaki T, Tanabe S, Azuma M et al. Propofol sedation with bispectral index monitoring is useful for endoscopic submucosal dissection: a randomized prospective phase II clinical trial. Endoscopy 2012; 44: $584-589$

[7] Nishizawa T, Suzuki H, Matsuzaki J et al. Propofol versus traditional sedative agents for endoscopic submucosal dissection. Dig Endosc 2014; 26: $701-706$

[8] Takimoto K, Ueda T, Shimamoto F et al. Sedation with dexmedetomidine hydrochloride during endoscopic submucosal dissection of gastric cancer. Dig Endosc 2011; 23: 176 - 181
[9] Kim N, Yoo YC, Lee SK et al. Comparison of the efficacy and safety of sedation between dexmedetomidine-remifentanil and propofol-remifentanil during endoscopic submucosal dissection. World J Gastroenterol 2015; 21: $3671-3678$

[10] Nonaka T, Inamori M, Miyashita T et al. Feasibility of deep sedation with the combination of propofol and dexmedetomidine hydrochloride for esophageal endoscopic submucosal dissection. Dig Endosc 2016; 28: $145-151$

[11] Cohen LB, Delegge MH, Aisenberg J et al. AGA Institute review of endoscopic sedation. Gastroenterol 2007; 133: 675 - 701

[12] Kidney Disease: Improving Global Outcomes (KDIGO) CKD Work Group. KDIGO 2012 clinical practice guideline for the evaluation and management of chronic kidney disease. Kidney Inter 2013; 3: 1-150

[13] Japanese Gastric Cancer Association. Japanese classification of gastric carcinoma: 3rd English edition. Gastric Cancer 2011; 14: 101-112

[14] Standards of Practice Committee of the American Society for Gastrointestinal Endoscopy. Lichtenstein DR, Jagannath $S$ et al. Sedation and anesthesia in $\mathrm{Gl}$ endoscopy. Gastrointest Endosc 2008; 68: 815-826

[15] Brignole M, Menozzi C, Del Rosso A et al. New classification of haemodynamics of vasovagal syncope: beyond the VASIS classification. Analysis of the pre-syncopal phase of the tilt test without and with nitroglycerin challenge. Vasovagal Syncope International Study. Europace 2000; 2: 66 - 76

[16] Bloor BC, Ward DS, Belleville JP et al. Effects of intravenous dexmedetomidine in humans. II. Hemodynamic changes. Anesthesiology 1992; 77: $1134-1142$

[17] Khan ZP, Ferguson CN, Jones RM. Alpha-2 and imidazoline receptor agonists. Their pharmacology and therapeutic role. Anaesthesia 1999; 54: $146-165$

[18] Shelly MP. Dexmedetomidine: a real innovation or more of the same? $\mathrm{Br}$ J Anaesth 2001; 87: 677-678

[19] Nishizawa T, Suzuki H, Sagara S et al. Dexmedetomidine versus midazolam for gastrointestinal endoscopy: a meta-analysis. Dig Endosc 2015; $27: 8-15$

[20] Venn RM, Bradshaw C], Spencer R et al. Preliminary UK experience of dexmedetomidine, a novel agent for postoperative sedation in the intensive care unit. Anaesthesia 1999; 54: 1136-1142

[21] American Society of Anesthesiologists. Task Force on Sedation and Analgesia by Non-Anesthesiologists. Practice guidelines for sedation and analgesia by non-anesthesiologists. Anesthesiology 2002; 96 : $1004-1017$ 